\title{
Advanced Myoelectric Control for Robotic Hand-Assisted Training: Outcome from a Stroke Patient
}

\author{
Zhiyuan Lu', Kai-yu Tong'2, Henry Shin ${ }^{1}$, Sheng Li' and Ping Zhou ${ }^{1,3 *}$ \\ ${ }^{1}$ Department of Physical Medicine and Rehabilitation, University of Texas Health Science Center at Houston, TIRR Memorial \\ Hermann Research Center, Houston, TX, USA, ${ }^{2}$ Division of Biomedical Engineering, Department of Electronic Engineering, \\ The Chinese University of Hong Kong, Hong Kong, Hong Kong, ${ }^{3}$ Guangdong Work Injury Rehabilitation Center, Guangzhou, \\ China
}

A hand exoskeleton driven by myoelectric pattern recognition was designed for stroke rehabilitation. It detects and recognizes the user's motion intent based on electromyography (EMG) signals, and then helps the user to accomplish hand motions in real time. The hand exoskeleton can perform six kinds of motions, including the whole hand closing/opening, tripod pinch/opening, and the "gun" sign/opening. A 52-year-old woman, 8 months after stroke, made $20 \times 2$-h visits over 10 weeks to participate in robot-assisted hand training. Though she was unable to move her fingers on her right hand before the training, EMG activities could be detected on her right forearm. In each visit, she took $4 \times 10$-min robot-assisted training sessions, in which she repeated the

OPEN ACCESS

Edited by:

Ayrton R. Massaro, Hospital Sirio-Libanes, Brazil

Reviewed by: Jun Yao,

Northwestern University, USA Michele Linda Callisaya, University of Tasmania, Australia

*Correspondence: Ping Zhou

ping.zhou.1@uth.tmc.edu

Specialty section: This article was submitted to Stroke, a section of the journal

Frontiers in Neurology

Received: 07 October 2016 Accepted: 03 March 2017 Published: 20 March 2017

Citation:

Lu Z, Tong KY, Shin H, Li S and Zhou P (2017) Advanced Myoelectric Control for Robotic Hand-Assisted

Training: Outcome from a Stroke

Patient.

Front. Neurol. 8:107.

doi: 10.3389/fneur.2017.00107 aforementioned six motion patterns assisted by our intent-driven hand exoskeleton. After the training, her grip force increased from 1.5 to $2.7 \mathrm{~kg}$, her pinch force increased from 1.5 to $2.5 \mathrm{~kg}$, her score of Box and Block test increased from 3 to 7 , her score of Fugl-Meyer (Part C) increased from 0 to 7 , and her hand function increased from Stage 1 to Stage 2 in Chedoke-McMaster assessment. The results demonstrate the feasibility of robot-assisted training driven by myoelectric pattern recognition after stroke.

Keywords: electromyography, myoelectric pattern recognition, hand exoskeleton, rehabilitation, case report

\section{INTRODUCTION}

Robot-assisted upper limb training is considered to be more efficient (1) and economic (2) than conventional therapy in neurorehabilitation. Controlling the robot with the user's own electromyography (EMG) signals connects the user's intended motion and his actual movements. It can therefore enhance therapeutic effects and promote motor learning (3-5). Various EMG-driven robots and exoskeletons have been developed for neurorehabilitation (6-8), primarily based on one-to-one mapping, which typically maps one channel of EMG signal to a corresponding single degree-offreedom (DOF) or variable such as speed and torque using a conventional "on-off" or proportional strategy. Robots based on such control strategy work well on training joints with only a few DOFs such as elbow and wrist. However, a human hand has up to $27 \mathrm{DOFs}(9)$ and is controlled by complex temporal and spatial coordination of multiple muscles. It is therefore not feasible to regain hand dexterity through conventional control strategies. Myoelectric pattern-recognition techniques have been developed to extract motion intentions from EMG signals $(10,11)$. The extracted intentions can then be used to control a multiple-DOF robot such as a prosthesis (12). Previous studies have also shown that motion intentions can still be extracted after neurological impairment (13-15). 
We therefore developed an intent-driven hand training system. The system employs an exoskeleton hand, which is controlled by myoelectric pattern recognition. As soon as the user's intention is detected (usually within $250 \mathrm{~ms}$ ), the system is able to assist to accomplish the intended motions (16).

\section{CASE REPORT}

\section{Subject}

A 52-year-old woman participated in this robotic hand-assisted training 8 months after stroke. She was right-handed before stroke and had hemiplegia on her right side after her stroke. She was able to walk independently with an ankle foot orthosis but had difficulties in moving her right arm. Her fingers were flexed naturally. She was unable to move any of the fingers on her right hand, but EMG signals were able to be recorded from her forearm. Her Fugl-Meyer score (Part A-D, max 66) was 16, with a 0 in Part $C$ (Hand, max 14). She had no pain when her whole hand was passively opened or closed. She did not receive any other hand or upper limb therapies while participating in this study. During her visits, she was able to understand and follow all the instructions.

\section{Exoskeleton Hand}

The exoskeleton hand, Hand of Hope (Rehab-Robotics, Hong Kong), was used in this study to help the subject move her hand (Figure 1). The exoskeleton hand has five individual fingers. Each finger is actuated by a linear actuator that can pull and push linearly. The mechanical design of the fingers converts these linear movements into the rotations of a virtual metacarpophalangeal (MCP) joint and a virtual proximal interphalangeal (PIP) joint. Both joints rotate together to help the hand perform closing and opening movements (7). The motion range is $55^{\circ}$ and $65^{\circ}$ for MCP and PIP joints, respectively. The subject's palm and five fingers are fixed to the exoskeleton hand with Velcro belts. Each finger can be bent or straightened individually by the exoskeleton hand. The exoskeleton hand stands on a brace, which also supports the

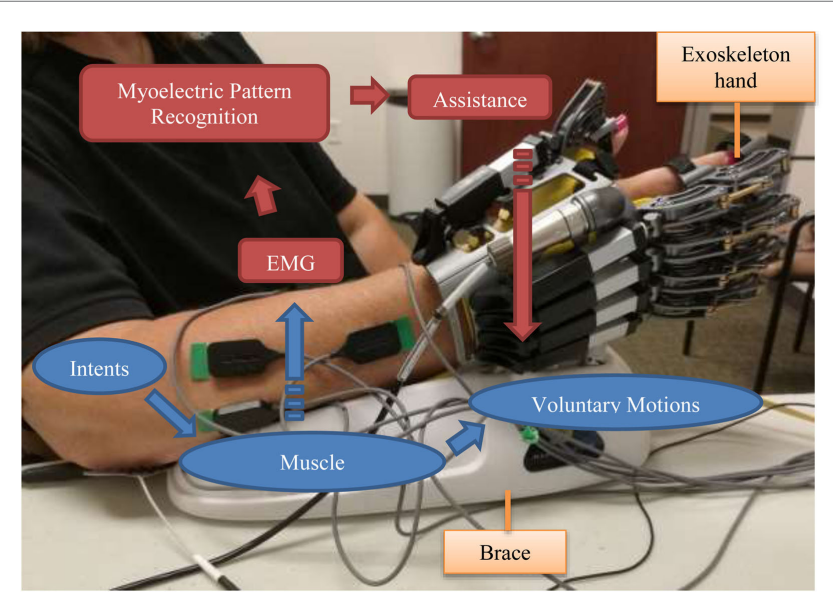

FIGURE 1 | Training with the exoskeleton hand driven by myoelectric pattern recognition. subject's forearm, so that the subject can be totally relaxed when attached to the exoskeleton. The exoskeleton hand used in this study can perform six different motion patterns, including hand closing ( $\mathrm{HC})$; hand opening ( $\mathrm{HO})$; thumb, index, and middle fingers closing (TIMC or tripod pinch); thumb, index, and middle fingers opening; middle, ring, and little fingers closing (MRLC or the "gun" sign); and middle, ring, and little fingers opening. The exoskeleton hand can perform HC, TIMC, or MRLC when it is open. However, after performing any one from these three patterns, it can only return to the original open status (e.g., there is no direct way from the "tripod pinch" to the "gun" sign).

Conventional EMG control of the device was applied in previous studies for training hand opening/closing function for stroke survivors $(17,18)$. In order to make all these six motion patterns available for the subject, a myoelectric pattern-recognition system was developed for this study to control the exoskeleton hand. This system is able to detect and recognize the subject's muscle activity patterns, indicate his/her intended hand motions, and then assist the patient in accomplishing these motions in real time. When the subject tries to perform a hand motion, EMG signals can be detected from those activated muscles. The myoelectric pattern-recognition system then extracts the motion intent from these EMG signals and maps the intent into control commands. The exoskeleton hand therefore performs the same motion as the subject's intent, so that the subject can accomplish the motion with both robotic assistance and his/her own participation.

\section{Protocol}

The subject made 20 visits (experiments) for the robot-assisted training, 2 visits per week. During the experiment, the subject was seated comfortably in a chair, next to a small height-adjustable side table. The exoskeleton hand was placed beside her on the table on her right side. Her right hand was fixed in the exoskeleton, and her forearm was placed on the brace (Figure 1). The exoskeleton was placed and locked on the brace. Therefore, the subject's right arm and hand could be totally relaxed instead of resisting gravity. The height of the table was adjusted to make the angle between her upper arm and her trunk about $45^{\circ}$, and the angle between her upper arm and her forearm about $90^{\circ}$. The subject was free to move her left hand. She was also allowed to move her right arm by moving the brace when she took breaks between two training sessions. Considering that the virtual palm was locked, the subject's right hand was always in a neutral position, and her forearm was never rotated even when she moved the brace. The brace would be moved to its initial position before another training session began.

Seven bipolar surface electrodes (Delsys 2.1) were attached on the subject's forearm using double-sided tapes, covering the first dorsal interossei, flexor digitorum superficialis, flexor digitorum profundus, extensor digitorum, abductor pollicis longus, extensor digiti minimi, and extensor pollicis longus muscles. The reference electrode was placed on the olecranon. The skin was cleaned using sterile alcohol wipes before electrodes were placed. EMG signals were acquired using a Bagnoli-8 EMG System (Delsys Inc., Boston, MA, USA), which amplified raw EMG signals 10,000 times and filtered the signals using a $20-450 \mathrm{~Hz}$ band pass filter. The acquired EMG signals were then input into a desktop 
running Windows 7 through a data acquisition device, USB-6221 (National Instruments Inc., Austin, TX, USA), which digitized the signals at $1,000 \mathrm{~Hz}$ with a 16 -bit resolution.

The digitized EMG signals were then analyzed by a myoelectric pattern-recognition program developed for this study, which aimed to extract motion intentions from multi-channel EMG signals. The analysis was based on the EMG signals recorded in the most recent $200 \mathrm{~ms}$ (named a processing window) and was performed every $100 \mathrm{~ms}$, so that the recognition result could be updated at $10 \mathrm{~Hz}$ (19), which is acceptable for real-time control. A motion detection algorithm was first performed to tell whether this window contained EMG signals that corresponded to the user's voluntary motions. It calculated the mean absolute value (MAV) (20) of the processing window. If the MAV was smaller than a given threshold (it was $80 \%$ of the average MAV of EMG signals recorded from this subject at her median force level), no further processing would be performed, and the recognition result was "no motion." Otherwise, a motion was considered to be detected because one or more muscles were active. Then, a pattern-recognition algorithm was performed, in which a support vector machine classifier (21) was applied to recognize motions based on a set of features including root mean square amplitude (20), fourth order auto regressive coefficients (22), and waveform length (20). The output of the classifier was then mapped onto control commands and sent to the hand exoskeleton.

Each visit included five sessions. In the first session, the subject repeated each motion pattern 15 times. Although she was unable to move her fingers, she was encouraged to try controlling the fingers. For each motion pattern, the subject was asked to imagine moving her fingers in the desired motion. The first $2 \mathrm{~s}$ of abovethreshold EMG signals were recorded before the exoskeleton subsequently provided assistance corresponding to the motion pattern. These EMG signals recorded in these 2-s periods were used to train the classifier. The next four sessions were all training sessions. In each session, the subject controlled the exoskeleton using her own motion intent. She was asked to try performing all of the six motion patterns and then to follow through with the exoskeleton hand while it moved through its full motion range. When the exoskeleton reached the final range, it stopped, and began waiting for another motion intent from the subject. The subject was therefore encouraged to perform the next motion right after the exoskeleton stopped. The subject was always free to choose any of the executable patterns, but sometimes the experimenter gave suggestions in order to balance the training amount of each motion pattern. Considering the subject's EMG signals were very weak, it was necessary for her to perform motions using about $70 \%$ of her maximal force in all these sessions. Consequently, she got fatigued quickly after 8-10 min training. In order to avoid fatigue, each training session was set to $10 \mathrm{~min}$ and could be terminated at any time after $8 \mathrm{~min}$. Moreover, she was given as much time as needed to rest between sessions. Therefore, each visit took about $2 \mathrm{~h}$, including approximately $40 \mathrm{~min}$ training.

\section{RESULTS}

Assessments, including grip force (using Jamar Plus + Digital Hand Dynamometer, Patterson Medical, Warrenville, IL, USA), pinch force (using PG-60 Pinch Gauges, B\&L Engineering, Santa Ana, CA, USA), Box and Block test (23), Fugl-Meyer (Part C only, ranging from 0 to 14), and Chedoke-McMaster (the Hand Stage only, ranging from 1 to 7) (24), were performed before and after the 20-visit training. Results are shown in Table 1. The results of both the grip force and the pinch force were the maximal readings from three measurements. The result of Box and Block test was the highest score of three trials. As to the Fugl-Meyer and Chedoke-McMaster assessment, only the handrelated score were reported because only hand function was trained. The average control accuracy of the 1st and the 20th visit was also calculated. The subject was requested to report every time when the exoskeleton hand performed a motion that was different from her intent. The control accuracy was calculated based on the number of wrong motions and the total number of motions.

After the training, the results of all the assessments were improved dramatically. The grip force and pinch force were almost doubled. She also regained some voluntary finger movements. These improvements were quantified by three functional assessments from different aspects. Before the training, she was not able to perform observable finger movements. Her Fugl-Meyer score (Part C) was 0 and Chedoke-McMaster stage was 1 because she failed to do all the tasks in these assessments. After the training, she could flex all her fingers in a small range, so that she was able to partially perform many of the tasks. For example, she was able to hold a pencil, though loosely. As a result, she obtained 1 point in each grip task in the part $\mathrm{C}$ of the Fugl-Meyer assessment except the task "flexion in interphalangeal joints and extension in MCP." Also because of these active motions, she met the criteria of Hand Stage 2 in the Chedoke-McMaster assessment. However, she did not get to Stage 3 because her range of motion was not greater than $50 \%$ plus that she did not have opposition to bring the thumb to the index finger. The same functional improvement increased her score in the Box and Block test. Because she was not able to open/close her hand before the training, she developed an alternative way to accomplish this task, which was to push a block into the space between her thumb and other fingers using arm movements. When she managed to push two corners of a block into her hand, she could pick up the block. Holding the block in hand was difficult given that her grip was weak and she was not holding the whole block. As a result, preventing dropping the block half way was more challenging for her, compared with releasing the block. After the training, she used the same way to move the blocks. Although the range of motion for $\mathrm{HO}$ was still not large enough to pick up or hold one block, it was easier for her to push the block in. And she could hold the block for a longer

TABLE 1 | Assessment results before and after the training.

\begin{tabular}{lcc}
\hline Tests & Pretreatment & Posttreatment \\
\hline Grip force $(\mathrm{kg})$ & 1.5 & 2.7 \\
Pinch force $(\mathrm{kg})$ & 1.5 & 2.5 \\
Box and Block & 3 & 7 \\
Fugl-Meyer (part C) & 0 & 7 \\
Chedoke-McMaster (hand) & 1 & 2 \\
Control accuracy (\%) & 75.0 & 76.9
\end{tabular}


time because of her increased grip force, so that she had more time to move the block over the barrier. The scores of her three trials after training were 4,7 , and 4 , respectively, while her highest score before training was 3 .

\section{DISCUSSION}

In the previous studies applying conventional control $(17,18)$, the exoskeleton was triggered when the EMG amplitude of the monitored muscle(s) exceeded a given threshold, while other muscles' activities were ignored. However, finger motions are generated by coordinating a series of muscles. Pattern-recognition algorithms were therefore introduced in this study in order to analyze the motion patterns of up to seven muscles. These algorithms also made it possible to control the hand exoskeleton in multiple DOFs and provided an approach to training fine motions and improving hand dexterity. Both the myoelectric pattern-recognition techniques and the robot-assisted training are safe. No adverse event was observed, and no discomfort was reported. Although fatigue was reported sometimes, it usually went away after a few minutes break.

This intent-driven control required the subject to be active during the training. This subject was able to activate her muscles though she could not perform finger motions. The real-time assistance from the exoskeleton gave her the feedback of muscle activities and helped her strengthen her motion patterns. Although the subject had severely impaired hand functions before the training (Hand Stage 1 according to the ChedokeMcMaster assessment), she still achieved 75\% control accuracy. Our algorithms recognized most of the subject's motion intents correctly, which assisted her in accomplishing these motions. Her hand function improved after the training, and all the assessments showed consistent improvements.

Although the training program for this subject demonstrates promising outcomes, a comprehensive evaluation of the effectiveness of the robotic hand-assisted training driven by myoelectric pattern recognition requires testing with a larger number of stroke subjects. We are aware that for a wide range of stroke patients with mild to severe impairment, some patients may not

\section{REFERENCES}

1. Lum PS, Burgar CG, Shor PC, Majmundar M, Van der Loos M. Robot-assisted movement training compared with conventional therapy techniques for the rehabilitation of upper-limb motor function after stroke. Arch Phys Med Rehabil (2002) 83(7):952-9. doi:10.1053/apmr.2001.33101

2. Wagner TH, Lo AC, Peduzzi P, Bravata DM, Huang GD, Krebs HI, et al. An economic analysis of robot-assisted therapy for long-term upperlimb impairment after stroke. Stroke (2011) 42(9):2630-2. doi:10.1161/ STROKEAHA.110.606442

3. Song R, Tong KY, Hu XL, Li L. Assistive control system using continuous myoelectric signal in robot-aided arm training for patients after stroke. IEEE Trans Neural Syst Rehabil Eng (2008) 16:371-9. doi:10.1109/ TNSRE.2008.926707

4. Lum PS, Burgar CG, Kenney DE, Vander Loos HF. Quantification of force abnormalities during passive and active-assisted upper-limb reaching movements in post-stroke hemiparesis. IEEE Trans Biomed Eng (1999) 46:652-62. doi:10.1109/10.764942

5. Hu XL, Tong KY, Song R, Zheng XJ, Leung WW. A comparison between electromyography-driven robot and passive motion device on wrist be suitable for such training due to lack of muscle activity or impaired muscle activity patterns $(25,26)$, while those stroke subjects who are able to generate muscle activity patterns and achieve reasonable accuracies can participate in the training program. In this regard, a pre-examination or assessment might be necessary to determine the stroke subjects who are able to control the exoskeleton hand with myoelectric pattern recognition, and who can benefit most from the robotic hand aided training.

\section{ETHICS STATEMENT}

This study was approved by the Committee for the Protection of Human Subjects (CPHS) of The University of Texas Health Science Center at Houston and TIRR Memorial Hermann (Houston, TX, USA). All procedures of the study were performed in accordance with the Declaration of Helsinki. The subject gave written and informed consent before the experimental procedures.

\section{AUTHOR CONTRIBUTIONS}

ZL performed experiment, analyzed data, and wrote the manuscript. KT developed the robotic exoskeleton hand and helped with data analysis. HS helped with experiment and data analysis. SL helped with data analysis and interpretation. PZ oversaw the study and helped with experiment, data analysis, and interpretation. All the authors read, revised, and approved the manuscript.

\section{ACKNOWLEDGMENTS}

The authors would like to thank Kathryn Nedley, OTR, OTD, for performing functional assessments and useful discussions.

\section{FUNDING}

This study was supported by American Heart Association (grant number 16GRNT29100012).

rehabilitation for chronic stroke. Neurorehabil Neural Repair (2009) 23:837-46. doi:10.1177/1545968309338191

6. Schabowsky CN, Godfrey SB, Holley RJ, Lum PS. Development and pilot testing of HEXORR: hand exoskeleton rehabilitation robot. J Neuroeng Rehabil (2010) 7:36. doi:10.1186/1743-0003-7-36

7. Ho NSK, Tong KY, Hu XL, Fung KL, Wei XJ, Rong W, et al. An EMG-driven exoskeleton hand robotic training device on chronic stroke subjects: task training system for stroke rehabilitation. IEEE Int Conf Rehabil Robot (2011) 2011:5975340. doi:10.1109/ICORR.2011.5975340

8. Heo P, Gu GM, Lee SJ, Rhee K, Kim J. Current hand exoskeleton technologies for rehabilitation and assistive engineering. Int J Precis Eng Manuf (2012) 13:807-24. doi:10.1007/s12541-012-0107-2

9. Lin J, Wu Y, Huang TS. Modeling the constraints of human hand motion. Proceedings Workshop on Human Motion. Washington, DC: IEEE (2000). p. 121-6.

10. Lu Z, Chen X, Li Q, Zhang X, Zhou P. A hand gesture recognition framework and wearable gesture-based interaction prototype for mobile devices. IEEE Trans Hum Mach Syst (2014) 44:293-9. doi:10.1109/THMS.2014.2302794

11. Oskoei MA, Hu H. Myoelectric control systems - a survey. Biomed Signal Process Control (2007) 2(4):275-94. doi:10.1016/j.bspc.2007.07.009 
12. Parker P, Englehart K, Hudgins B. Myoelectric signal processing for control of powered limb prostheses. JElectromyogr Kinesiol (2006) 16:541-8. doi:10.1016/j.jelekin.2006.08.006

13. Liu J, Zhou P. A novel myoelectric pattern recognition strategy for hand function restoration after incomplete cervical spinal cord injury. IEEE Trans Neural Syst Rehabil Eng (2013) 21:96-103. doi:10.1109/TNSRE.2012.2218832

14. Zhang X, Zhou P. High-density myoelectric pattern recognition toward improved stroke rehabilitation. IEEE Trans Biomed Eng (2012) 59(6):1649-57. doi:10.1109/TBME.2012.2191551

15. Liu J, Li X, Marciniak C, Rymer WZ, Zhou P. Extraction of neural control commands using myoelectric pattern recognition: a novel application in adults with cerebral palsy. Int J Neural Syst (2014) 24(7):1450022. doi:10.1142/ S0129065714500221

16. Lu Z, Chen X, Zhang X, Tong K, Zhou P. Real-time control of an exoskeleton hand robot with myoelectric pattern recognition. Int J Neural Syst (2016) 27:1750009. doi:10.1142/S0129065717500095

17. Hu XL, Tong KY, Wei XJ, Rong W, Susanto EA, Ho SK. The effects of post-stroke upper-limb training with an electromyography (EMG)-driven hand robot. J Electromyogr Kinesiol (2013) 23:1065-74. doi:10.1016/j.jelekin.2013.07.007

18. Rong W, Tong KT, Hu XL, Ho SK. Effects of electromyography-driven robot-aided hand training with neuromuscular electrical stimulation on hand control performance after chronic stroke. Disabil Rehabil Assist Technol (2015) 10:149-59. doi:10.3109/17483107.2013.873491

19. Li Z, Wang B, Sun F, Yang C, Xie Q, Zhang W. sEMG-based joint force control for an upper-limb power-assist exoskeleton robot. IEEE J Biomed Health Inform (2014) 18:1043-50. doi:10.1109/JBHI.2013.2286455

20. Chowdhury RH, Reaz MB, Ali MA, Bakar AA, Chellappan K, Chang TG. Surface electromyography signal processing and classification techniques. Sensors (Basel) (2013) 13(9):12431-66. doi:10.3390/s130912431
21. Chang C, Lin C. LIBSVM: a library for support vector machines. ACM Trans Intell Syst Technol (2011) 27:1-27. doi:10.1145/1961189.1961199

22. Khokhar ZO, Xiao ZG, Menon C. Surface EMG pattern recognition for real-time control of a wrist exoskeleton. Biomed Eng Online (2010) 9:41. doi:10.1186/1475-925X-9-41

23. Mathiowetz V, Volland G, Kashman N, Weber K. Adult norms for the Box and Block test of manual dexterity. Am J Occup Ther (1985) 39(6):386-91. doi:10.5014/ajot.39.6.386

24. Gowland CA. Staging motor impairment after stroke. Stroke (1990) 21:19-21.

25. Cesqui B, Tropea P, Micera S, Krebs HI. EMG-based pattern recognition approach in post stroke robot-aided rehabilitation: a feasibility study. J Neuroeng Rehabil (2013) 10(1):75. doi:10.1186/1743-0003-10-75

26. Lee SW, Wilson KM, Lock BA, Kamper DG. Subject-specific myoelectric pattern classification of functional hand movements for stroke survivors. IEEE Trans Neural Syst Rehabil Eng (2011) 19(5):558-66. doi:10.1109/ TNSRE.2010.2079334

Conflict of Interest Statement: The authors declare that the research was conducted in the absence of any commercial or financial relationships that could be construed as a potential conflict of interest.

Copyright (C) 2017 Lu, Tong, Shin, Li and Zhou. This is an open-access article distributed under the terms of the Creative Commons Attribution License (CC BY). The use, distribution or reproduction in other forums is permitted, provided the original author(s) or licensor are credited and that the original publication in this journal is cited, in accordance with accepted academic practice. No use, distribution or reproduction is permitted which does not comply with these terms. 